\title{
THE ESTIMATION OF URIC ACID
}

\author{
BY ITS BRDUCING ACTION UPON THE
}

\section{AMMONIATED CUPRIC TEST.}

\author{
BD \\ F. W. PAVY, M.D., F.R.S., \\ PHYSIOISN TO, $\triangle$ SD IROTORFR ON MEDIOTNR $\triangle T$, GUY'S HOSPITAL.
}

(Received February 10th-Read March 23rd, 1880.

Throdar uric acid more or less serious trouble is not unfrequently produced. A natural constituent of the urine to a limited extent, it possesses more important pathological than physiological bearings. It is its appearance as a deposit which gives rise to harm, and to obviate this is a task often placed before us as medical practitioners. The task may be readily enough accomplished by the administration of certain agents which lead to the formation of a soluble product, but at present our knowledge does not extend much beyond this, and when the agent is discontinued, it but too often happens that the deposit again shows itself.

As a means of studying the various conditions which tell favorably and unfavorably upon the uric acid diathesis, and likewise as a guide during the management of a case 
of the occurrence of the deposit, the information afforded by the quantitative determination of the uric acid voided forms an item of the first importance. It is to the consideration of the manner in which this determination may be satisfactorily effected, that this communication is devoted.

The methods hitherto employed for the estimation of uric acid cannot be spoken of as suitably meeting the requirements. Both as regards precision and convenience or facility of application, they leave much to be desired. The simple collection of the crystals which settle from a specimen of urine after the addition of an acid constitutes a very unsatisfactory process, as, on account of the partial solubility which uric acid possesses, only a portion of it present becomes thereby estimated. A more accurate plan is that of evaporating a given amount of urine to dryness, washing the residue with alcohol, and then treating it with dilute hydrochloric acid. In this way the uric acid is separated from the other constituents of the urine, and the quantity determined by weighing. This process, from the time it occupies, and from the manipulative conveniences required, is not adapted for general clinical use, nor is it altogether free from affording a lower estimate than should be yielded.

The process which it is the object of this communication to bring under the notice of the Society is one by which the estimation of uric acid may be effected not only speedily and accurately, but in such a manner as to be susceptible of being carried out by the medical practitioner.

It is well known that uric acid possesses, like glucose, the property of exerting a reducing action upon the oxide of copper. With the ordinary copper test this property does not admit of being turned to account for the estimation of uric acid in urine, as it does in the case of sugar. In the ammoniated copper test, however, which I have recently introduced and am employing with such a satisfactory result for the quantitative determination of sugar, 
not only have we an agent which is available for the estimation of sugar, but also for that of uric acid.

The test in question was described by me in communications to the Royal Society published in the 'Proceedings' (vol. xxviii, p. 260, and vol. xxix, p. 272). It is prepared by adding ammonia to the ordinary cupric test solution, the effect of which is to exert a solvent action upon the reduced oxide formed when the reagent is employed. Reduction occurs under the influence of the reducing agent, whether sugar or uric acid, but instead of the reduced oxide falling as an orange-coloured precipitate, it is held in solution by the ammonia in a colourless state, the change noticeable being the gradual removal of the blue colour belonging to the original liquid as reduction proceeds.

The instructions for preparing the liquid are as follows:

Let in the first place the ordinary cupric test solution be made according to the subjoined formula.

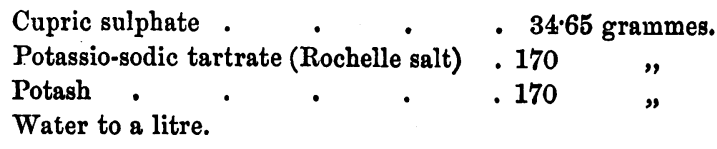

For the ammoniated liquid, 120 c.c. of this test solution are mixed with 300 c.c. of strong ammonia (sp. gr. 0.880), and water added so as to bring the volume to a litre.

The liquid thus produced is prepared for sugar estimation, and is adapted in strength to give an even numbered expression of value. The reducing power of uric acid bearing no correspondence with that of sugar, the value in uric acid presents itself as a fractional number. The strength might, of course, if desired, be so arranged as to correspond with any given amount of uric acid, but it seems scarcely worth while for the sake of a few figures in calculation, to provide an additional liquid for use.

The test is employed in the same way as for the estimation of sugar. Twenty c.c. of the ammoniated liquid are placed in a flask, which is affixed to a cork suspended by a piece of vulcanised tubing from a burette containing 
the product to be examined. The flame either of a spirit lamp or gas having been applied underneath the flask, its contents are brought to a boiling point, and the liquid in the burette is then dropped in under the control of a clip placed on the vulcanised tubing, until all trace of blue colour is just made to disappear. To obviate the inconvenience arising from the dispersion of the evolved ammonia into the air, it may be conducted by a piece of vulcanised tubing into a vessel of water. The end of the tubing which dips into the water being plugged at its extremity, and having a transverse slit through three fourths of its extent just above, supplies a valvular arrangement which permits of the escape of air and ammonia vapour from the flask, but prevents the sucking back of water with the sudden condensation that is liable to occur should it happen that the liquid is run in a little quickly from the burette. Messrs. Griffin, of Garrick Street, Covent Garden, supply the required apparatus.

Having at the outset learnt that in the application of the liquid for the estimation of uric acid the point of complete decoloration was easily susceptible of discrimination, and that uniformity was recognisable in the result, it next became necessary (in order that quantitative expressions might be given) to ascertain the precise reducing capacity appertaining to the principle.

Solutions of various strengths that had been prepared from carefully purified uric acid were submitted to trial, and the results obtained were such as to very closely agree with the formula representing three atoms of oxide of copper as reduced by one atom of uric acid. Actual observation, for instance, gave 0.03747 gramme of uric acid as the quantity taken to reduce 40 c.c. of the ammoniated cupric liquid. Theoretically, upon the basis of one atom of uric acid reducing three atoms of cupric oxide, the quantity would stand at 0.03732 gramme. From the close accord thus existing, I think the theoretical relation. ship mentioned may be justifiably accepted as the expression to be adopted. 
A. Burette containing the diluted urine.

B. Flask containing the ammoniated cupric test.

c. Clip to regulate the flow of the diluted urine into the flask.

D. Stand.

E. Spirit lamp.

F. Vulcanised tube to conduct the evolved ammonia into water contained in the ressel (G).

ז. Valve in the vulcanised tubing to prevent the sucking back of the water into the flask $\mathrm{B}$.

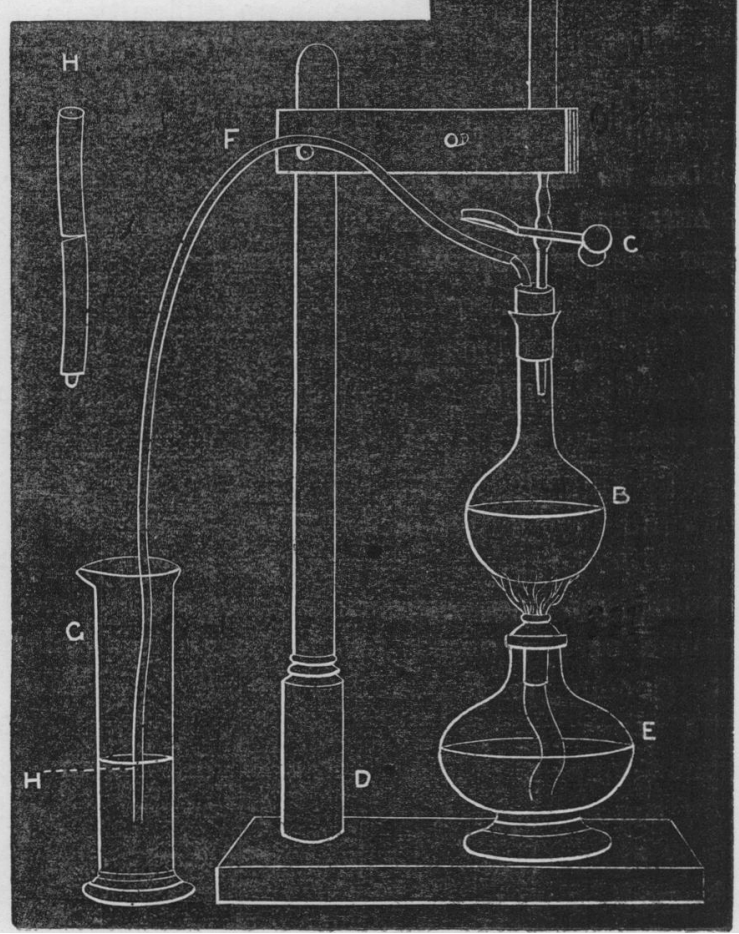


Reckoning, then, that one atom of uric acid reduces three atoms of oxide of copper, 20 c.c. of the ammoniated test will stand equivalent to 0.01866 gramme of uric acid.

In the application of the test to the determination of uric acid in nrine, 20 c.c. of it constitutes a convenient quantity to be taken, and experience shows that it is desirable to add after its introduction into the flask an equal bulk of water. This dilntion diminishes the interference produced by the colour of the urine, and with a specimen of urine of arerage concentration it is eren adrisable to dilute this with a corresponding bulk of water before placing it in the burette.

In this state it is found that somewhere about 8 or 10 c.c. of liquid are required to decolorise the 20 c.c. of the cupric test.

With a little experience there is no difficulty in determining when the required removal of blue colour has been effected, although the point of completion is not so sharply defined as in the estimation of sugar, on account of a slightly yellowish tint becoming developed during the operation performed. The contents of the burette must be dropped in till no sign of blue or green is left.

The actual determination of the uric acid in a specimen of urine is effected by taking the reducing power before and after precipitation with acetate of lead. The reducing action before the addition of acetate of lead is due partly to uric acid and partly to the small amount of sugar natarally present in urine. It is doubtful if there is any other body worthy of consideration to exert any sensible reducing effect.

The reducing power, expressed as uric acid, of one portion of the urine, is first determined in the ordinary way. Fifty c.c. of another portion of the urine are then taken and treated with 5 c.c. of a cold saturated solution of acetate of lead. It is necessary that enough acetate of lead should be added to secure complete precipitation, but it is desirable that there should not be a large excess left in solution. Unless the urine is very concentrated, the 
quantity of acetate of lead recommended will be found to be sufficient. The precipitate having been separated by filtration, the filtrate is treated with a small fragment of caustic potash, which first throws down the oxide of lead from the surplus acetate, and then redissolves it. The reducing power, again expressed as uric acid, of the urine thus operated upon is now taken, and to the figures yielded an addition of one tenth is made in order to supply the correction required for the dilution with the lead solution.

As an illustration, let it be supposed that 20 c.c. of the ammoniated cupric test, which are equivalent to 0.1866 grammes of uric acid, are placed in the flask and, after being brought to a state of ebullition, that the urine diluted with an equal volume of water is allowed to run into it from the burette. If we assume that 10 c.c. (equivalent to 5 c.c. of urine) are required to effect decoloration, the amount of reducing power, represented as uric acid, is $\frac{\cdot 01866 \times 1000}{5 \cdot 0}$, or $\frac{18 \cdot 66}{5}$, or 3.732 per 1000 .

This completes one step of the operation, and it gives the total reducing power (only a portion of which, however, is due to uric acid) of the urine. Let it be next supposed that 50 c.c. of the urine have been taken and mixed with 5 c.c. of the solution of acetate of lead, and that 7.5 c.c. of the filtrate are required to decolorise the 20 c.c. of the cupric test. We have here the reducing power of the urine minus that of its uric acid, which has been precipitated by the lead solution; and after making the necessary correction for dilution with the lead solution and working out the second calculation, the required data are furnished for showing the amount of uric acid that is present. The second calculation stands as follows:$\frac{.01866 \times 1000}{7 \cdot 5}$, or $\frac{18 \cdot 66}{7 \cdot 5}$, or $2 \cdot 488$ per 1000 , and correcting for the dilution $2 \cdot 488+0 \cdot 248=2 \cdot 736$ per 1000 .

Having learnt that the total reducing power was $3 \cdot 732$ per 1000 , and the reducing power after the precipitation of 
the uric acid $2 \cdot 736: 0.996$ per 1000 is given as the amount of uric acid present.

To ascertain if reliance could be placed upon the uric acid present being revealed by the method of procedure described, weighed quantities of it were added to specimens of urine and the estimation undertaken. The following results show that a close accord between the amount of uric acid added and that indicated was obtained.

\begin{tabular}{rcccccccc} 
& \multicolumn{3}{c}{$\begin{array}{c}\text { Uric acid found } \\
\text { before the addition } \\
\text { of uric acid, } \\
\text { per 1000. }\end{array}$} & $\begin{array}{c}\text { Uric acid found } \\
\text { after the addition } \\
\text { of uric aoid, } \\
\text { per 1000. }\end{array}$ & $\begin{array}{c}\text { Uric acid indicated } \\
\text { as having been } \\
\text { added per } \\
1000 .\end{array}$ & $\begin{array}{c}\text { Uric acid } \\
\text { actually } \\
\text { added, } \\
\text { per } 1000 .\end{array}$ \\
Obs. I & $\ldots$ & 0.597 & $\ldots$ & 1.194 & $\ldots$ & 0.597 & $\ldots$ & 0.600 \\
" II & $\ldots$ & 0.168 & $\ldots$ & 1.474 & $\ldots$ & 1.206 & $\ldots$ & 1.200 \\
" III & $\ldots$ & 0.653 & $\ldots$ & 2.649 & $\ldots$ & 1.996 & $\ldots$ & 2.000 \\
" IV & $\ldots$ & 0.653 & $\ldots$ & 1.613 & $\ldots$ & 0.960 & $\ldots$ & 1.000
\end{tabular}

A satisfactory illustration was also afforded by these observations of the complete precipitation of the uric acid that is effected by the acetate of lead. The filtrate from the lead precipitate belonging to the specimen in which uric acid had been added, presented no essential difference in reducing power from the corresponding specimens to which no uric acid had been added.

These observations suffice to show that whatever uric acid is present is revealed through the difference existing between the reducing action before and after precipitations with acetate of lead, but they do not prove that the difference is wholly attributable to uric acid. There might be something present besides uric acid precipitable by acetate of lead, and if so, it would be reckoned as uric acid. To ascertain whether the existence of such a source of fallacy is to be apprehended, observations were conducted, in which the results yielded by the process described were checked by the actual collection and subsequent determination of the uric acid.

For these check observations to be of any value, the method of procedure adopted must be secure against any loss of uric acid. After considerable attention, and trials made with added known quantities of uric acid, the fol- 
lowing is the plan I have been led to have recourse to. Fifty c.c. of urine are treated with acetic acid and evaporated to dryness. The employment of hydrochloric acid is avoided on account of the coloured product which is developed by it. The dried residue containing the uric acid in a liberated state is extracted and well washed with spirit. On account of its insolubility in spirit the uric acid is left in conjunction with some saline matter, which does not interfere with the subsequent part of the analysis. Instead of employing a filter paper, the insoluble residue is best collected upon an asbestos plug introduced into the neck of a funnel, and the plug and residue, when the washing is completed, are transferred to a beaker and treated with a weak solution of potash. This dissolves the uric acid, and the solution is separated by filtration, and brought to the volume of the urine started with. The estimation of the uric acid thus isolated is effected volumetrically with the cupric solution, and on account of this plan of estimation being adopted, the complete purification from saline matter (necesssary where weighing which has hitherto constituted the means of determination had recourse to) is not called for. In this method of procedure the employment of an aqueous liquid in washing is dispensed with, which is a material item, in consequence of the slight solvent action upon the uric acid it is capable of exerting.

Looking at the nature of this process, there does not appear to be any room for questioning that the results furnished by it may be taken as representative of uric acid; and, that it is susceptible of being worked so as to give a close approach to absolute accuracy is proved by the results derived from the observations which have been conducted upon specimens of urine to which known quantities of uric acid were added. In these observations the amount of uric acid was first determined in the urine itself by the process in question. Uric acid was then added and the estimation again undertaken. Subjoined are the figures yielded in three sets of observations.

vOL. LXIII. 
Estimation effected by the separation of the uric acid and its subsequent determination where known quantities had been added.

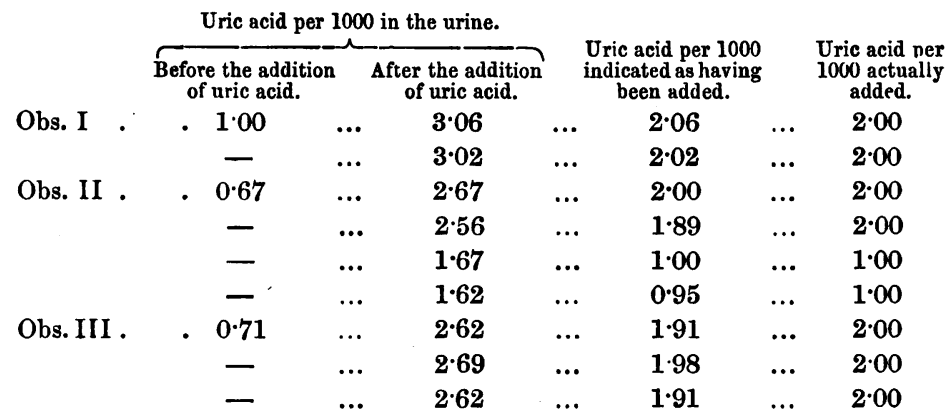

Having learnt that reliance may be placed upon the process of separation and determination which has been described, the means are supplied for testing the accuracy of the process which forms the special subject of this communication. The two processes have been applied to various specimens of urine, and, as the following tabulated representation shows, a close accord is noticeable in the respective results obtained. With this evidence before us, sufficient grounds are afforded to justify confidence being placed in the estimation founded upon the reducing power displayed before and after precipitation with acetate of lead.

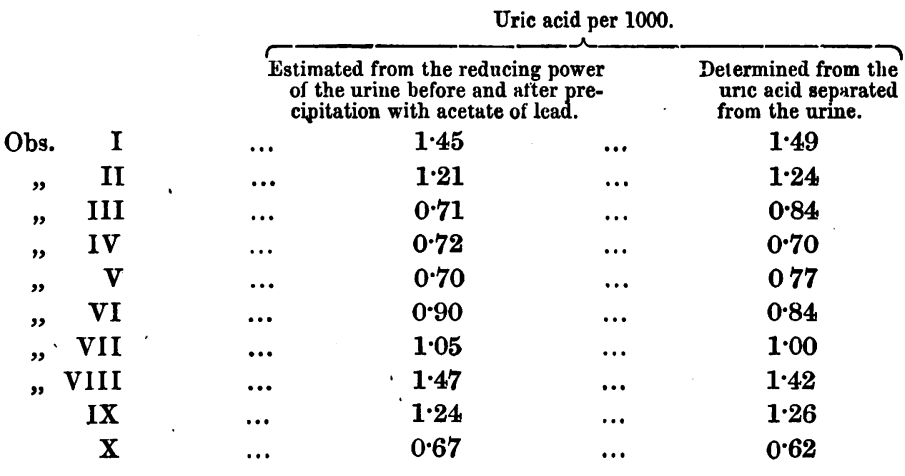


This completes what I have to say about the process I have described for the estimation of uric acid. The next matter to deal with is the results obtained by its application to the urine under various conditions of health and disease. This is a matter of some magnitude, which I must reserve for the subject of a future communication, but I may here state that, according to the observations I have conducted, the figures that have been hitherto given as representing the amount of uric acid present will have to undergo considerable alteration. 